\title{
ARTICLE \\ Rap1-mediated nuclear factor-kappaB (NF- $\kappa$ B) activity regulates the paracrine capacity of mesenchymal stem cells in heart repair following infarction
}

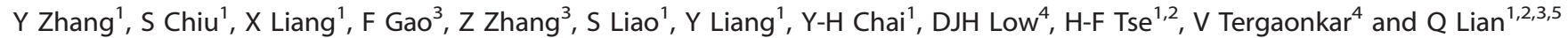

Paracrine effect is the major mechanism that underlies mesenchymal stem cells (MSC)-based therapy. This study aimed to examine how Rap1, telomeric repeat-binding factor 2-interacting protein 1 (Terf2IP), which is a novel modulator involved in the nuclear factor-kappaB (NF- $\kappa$ B) pathway, regulates the paracrine effects of MSC-mediated heart repair following infarction. NF- $\kappa B$ activity of stromal cells was increased by Rap1 as measured by pNF- $\kappa B$-luciferase reporter activity, and this was abolished by lkB-dominantnegative protein. Knockdown of Rap1 with shRap1 resulted in diminished translocation of p65-NF- $k$ B from the cytoplasm to nuclei in response to tumor necrosis factor- $a$ (TNF- $a$ ) stimulation. Compared with BM-MSCs, Rap $1^{-/-}-$BM-MSCs displayed a significantly reduced ratio of phosphorylated NF- $\kappa$ B to NF- $\kappa$ B-p65 and of Bax to Bcl-2, and increased resistance to hypoxia-induced apoptosis by the terminal deoxynucleotidal transferase-mediated dUTP nick end labeling (TUNEL) assay. In contrast, re-expression of Rap1 in $\mathrm{Rap}^{-/-}$-BM-MSCs resulted in loss of resistance to apoptosis in the presence of hypoxia. Moreover, absence of Rap1 in BM-MSCs led to downregulation of NF-KB activity accompanied by reduced pro-inflammatory paracrine cytokines TNF- $a$, IL (interleukin)- 6 and monocyte chemotactic protein-1 in Rap $1^{-/-}$-BM-MSCs compared with BM-MSCs. The apoptosis of neonatal cardiomyocytes (NCMCs) induced by hypoxia was significantly reduced when cocultured with Rap $1^{-/-}$-BM-MSC hypoxic-conditioned medium (CdM). The increased cardioprotective effects of Rap $1^{-1-}$-BM-MSCs were reduced when Rap $1^{-1-}$-BM-MSCs were reconstituted with Rap1 re-expression. Furthermore, in vivo study showed that transplantation of Rap1 ${ }^{-/-}$-BM-MSCs significantly improved heart function, decreased infarct size, prevented cardiomyocyte apoptosis and inhibited inflammation compared with controls and BM-MSCs $(P<0.01)$. This study reveals that Rap1 has a critical role in the regulation of MSC paracrine actions. Compared with BM-MSCs, Rap1 ${ }^{-/-}-$BM-MSCs decreased NF-KB sensitivity to stress-induced pro-inflammatory cytokine production and reduced apoptosis. Selective inhibition of Rap1 in BM-MSCs may be a novel strategy to enhance MSC-based therapeutic efficacy in myocardial infarction.

Cell Death Discovery (2015) 1, 15007; doi:10.1038/cddiscovery.2015.7; published online 24 August 2015

\section{INTRODUCTION}

Recent developments in stem cell biology to prevent or treat heart failure have moved from experimental research to clinical trials using different types of adult stem cells, such as adult bone marrow stem cells or mesenchymal stem cells (MSCs). ${ }^{1,2}$ Despite the promising results observed in preclinical and clinical studies, the potential mechanisms underlying stem cell-based therapy have not been fully classified. Accumulating evidence demonstrates that apart from transdifferentiation, it is the paracrine effects of MSCs that are predominately responsible for cardiac repair. ${ }^{3}$ Indeed, the exact extent to which these cells transdifferentiate into new cardiomyocytes to improve heart function remains highly controversial. ${ }^{4,5}$ There is increasing recognition that MSCs produce a variety of cytokines that can directly promote cell survival and regulate inflammation following myocardial infarction (MI). ${ }^{3,6}$ Various cytokines and growth factors have been investigated in different tissue-derived MSCs., ${ }^{7,8}$ Some cytokines and growth factors have been shown to be critical for cardiac protection, for example, basic fibroblast growth factor, vascular endothelial growth factor, stromal-derived factor, 9,10 secreted frizzled related protein, ${ }^{11}$ interleukin-10 (IL-10) ${ }^{12}$ and metalloproteinase-9. ${ }^{13}$ In contrast, some cytokines and factors produced from MSCs, including tumor necrosis factor- $a$ (TNF- $a$ ) and interleukin-6 (IL-6), ${ }^{14}$ are harmful for heart recovery. As a result, injection of MSCs or total MSC paracrine factors without optimization may limit their therapeutic efficacy. Therefore, optimization of MSCs before transplantation to maximize cell survival and beneficial paracrine factors is important. Nevertheless, the potential mechanism underlying regulation of MSC secretion is poorly understood and identification of the key molecules that govern MSC secretion to protect against heart ischemia injury remains urgently to be addressed.

Rap1 (Trf2IP), a telomeric repeat-binding factor 2-interacting protein 1 (Terf2IP), is a novel modulator involved in the Nuclear factor-kappaB (NF-KB) pathway. ${ }^{15,16}$ Many telomeric proteins apart from Rap1 including telomerase are now known to regulate inflammation through NF-KB signaling. ${ }^{17,18}$ It has been documented that NF-KB family factors have important roles in the

\footnotetext{
'Division of Cardiology, Department of Medicine, The University of Hong Kong, Hong Kong SAR, China; ${ }^{2}$ Research Centre of Heart, Brain, Hormone, and Healthy Aging, Li Ka Shing Faculty of Medicine, The University of Hong Kong, Hong Kong SAR, China; ${ }^{3}$ Department of Ophthalmology, Li Ka Shing Faculty of Medicine, The University of Hong Kong, Hong Kong SAR, China; ${ }^{4}$ Institute of Molecular and Cellular Biology, Biopolis, Singapore and ${ }^{5}$ Shenzhen University Health Science Center, Shenzhen, China. Correspondence: Q Lian (qzlian@hkucc.hku.hk)
}

Received 3 June 2015; accepted 5 June 2015; Edited by G Melino 
regulation of mitochondrial ROS/bioenergy, DNA replication, cell survival and inflammation in many cell types, ${ }^{19-22}$ including $\mathrm{MSCs}^{23}$ Prior studies have also demonstrated that NF- $K \mathrm{~B}$ activation mediates cytokine/growth factor secretion by MSCs. ${ }^{24}$ Given the multifaceted effects of NF- $K B$ activity on MSCs, efforts to identify important regulators (s) that modulate specificity in the functioning of the NF- $K B$ signal pathway is a major challenge. Use of a genetic engineering will provide a greater understanding of the roles and mechanisms of specific factors that modulate NF- $\kappa B$ activation in MSC-mediated cardioprotection. It also has been reported that MSCs can secrete a variety of cytokines to regulate inflammation and enable cardiac repair posttransplantation. ${ }^{25}$ Hence, to determine whether Rap1 can regulate MSC paracrine factors, which thereby impinges on cardiac repair, we designed this study. In this study, we proposed that inhibition of Rap1 can decrease pro-inflammatory factors secreted by MSCs, enhance cell survival and thus improve its therapeutic effects in MI. In this study, we showed that Rap $1^{-1-}$-BM-MSCs have a better therapeutic efficacy than wild-type (WT) BM-MSCs for cardiac repair post $\mathrm{Ml}$ in mice. The greater therapeutic potential of Rap $1^{-1}$-BM-MSCs for cardiac repair is not only attributed to the higher cell survival post-transplantation, but also to a reduced secretion of pro-inflammatory cytokines. Our results may prompt the development of new therapeutic strategies to enhance MSCbased therapy in MI.

\section{RESULTS}

Characterization of BM-MSCs and Rap1 $1^{-/-}$-BM-MSCs

As shown in Figure 1a, FACS analysis demonstrated that control BM-MSCs and Rap1 $1^{-/-}$-BM-MSCs have similar surface markers that are defined in BM-MSCs, that is, Sca (+), CD90 (+), CD105 (+), CD45 $(-)$ and CD34 (-; Figure 1a). Oil red staining showed that adipogenesis was highly observed in $>80 \%$ of BM-MSCs and Rap $^{-1-}$-BM-MSCs (Figure 1bi). Alcian Blue staining showed that chondrogenesis was efficient with $>80 \%$ cells for calcium deposition (Figure 1bii). Alizarin Red staining revealed that BM-MSCs and Rap1 $1^{-1-}$-BM-MSCs could differentiate into osteogenic cells (Figure 1biii). In addition, western blot analysis showed that Rap1 was detected in BM-MSCs, but not in Rap1 ${ }^{-/-}$-BM-MSCs (Figure 1C), indicating the total deletion of Rap1 from Rap1 ${ }^{-/-}-\mathrm{BM}-\mathrm{MSC}$.

\section{Activation of NF- $\kappa$ B transcriptional activity by Rap 1}

In order to address the direct relationship between NF- $K B$ and Rap1, the activation of NF- $k$ B transcriptional activity by Rap1 was examined. A reporter gene construct carrying NF-KB-Luc was used to report NF- $K B$ activity. This construct contains an NF- $K B$ enhancer element located upstream of the secreted luciferase gene. Binding of transcription factors to the NF- $\kappa \mathrm{B}$ enhancer element allows luciferase (tLuc) to be expressed and secreted into the surrounding medium (Catalog No. 631743; Clontech Laboratories, Mountain View, CA, USA). As shown in Figure 2a, HeLa cells were transfected with indicated plasmids. Groups $\mathrm{i}$ and iii received WT reporter constructs $p$ NF- $\kappa B-L u c$, whereas group ii received delta NF- $K B-L u c$. All groups received pSV-RLuc. The amount of expression plasmid for Rap1 (i and ii) and lkB-dominant-negative (iii) was progressively increased as indicated. Relative luciferase activity represents firefly luciferase activity recovered from pNF- $\kappa B$-Luc or pdelta $\kappa B$-Luc normalized to Renilla luciferase activity recovered from pSV-RLuc (Figure 2a). The results presented showed that the activity of NF- $\kappa$ B transcriptional activity was activated by Rap1.

Next, the relationship of IKKY and Rap1 was examined. As shown in Figure 2b, HEK293T cells were transfected with expression plasmids for Flag-tagged Rap1 (lanes 3 and 4), HA-tagged IKKY (lanes 2 and 4). All groups received His-tagged ubiquitin expression plasmid. His-tagged ubiquitin conjugates were pulled down. The ubiquitinated Rap1 and IKKY were detected using western blot analysis with anti-Flag (Figure 2bi) and anti-HA (Figure 2biii), respectively. The input lysates were also probed for anti-Flag and anti-HA to detect Rap1 and IKK $\gamma$, respectively (Figure $2 \mathrm{bii}$ and iv). These results show the IKKYmediated ubiquitination of Rap1.

Immunostaining results demonstrated that NF- $K B$ translocated from the cytoplasm to nuclei when HeLa cells were stimulated with TNF- $a$ (Figure 2ci and ii). Nonetheless, translocation of NF- $\kappa$ B-p65 from the cytoplasm to nuclei responding to TNF-a stimulation was diminished by knocking down Rap1 with shRap 1 (Figure 2ciii and iv). These results show that the absence of Rap1 negatively regulates activation of NF- $K \mathrm{~B}$.

\section{Rap1 $1^{-/-}$-BM-MSCs are more tolerant than BM-MSCs} to hypoxia-induced apoptosis

Apoptosis of Rap $1^{-/-}$-BM-MSCs and BM-MSCs under normoxic or hypoxic conditions was examined. As shown in Figures $3 a$ and $b$, the apoptotic rate of Rap1 $1^{-/-}$-BM-MSCs and BM-MSCs under normoxia was similar and very low (Figure 3b, $2.55 \pm 0.59 \%$ versus $1.7 \pm 0.52 \% ; \quad P>0.05)$. Nonetheless, compared with normoxia, the apoptotic rate was significantly increased under hypoxic conditions. Notably, compared with BM-MSCs, the apoptotic rate of Rap1 $1^{-1-}$-BM-MSCs under hypoxia was much lower (Figure 3b, $17.7 \pm 1.2 \%$ versus $8.1 \pm 0.87 \%$; $P<0.01$ ), suggesting that $\operatorname{Rap}^{-i-}$-BM-MSCs have superior resistance to the challenge of hypoxia.

As shown in Figure 3c, western blot analysis results demonstrated that hypoxia significantly increased the ratio of $p-N F-\kappa B /$ NF- $\kappa$ B-p65 and the ratio of Bax/Bcl-2 in Rap $1^{-1-}-\mathrm{BM}-\mathrm{MSCs}$ and BMMSCs. Notably, compared with BM-MSCs, NF- $K B$ activation and the levels of $\mathrm{Bax} / \mathrm{BCl}-2$ were greatly reduced in $\mathrm{Rap}^{-1-}$-BM-MSCs under conditions of hypoxia (Figure 3c).

To further understand the role of Rap1 in the regulation of MSCs against hypoxia, deficiency of Rap $1^{-1-}$-BM-MSCs was replenished with Rap1 re-expression using a lentivirus system. As shown in Figure 3di, Rap1 was detected in Rap1-Rap $1^{-1-}$-BM-MSCs but not in Rap $1^{-/}-$BM-MSCs. Under hypoxic challenge, apoptosis of Rap1Rap1 ${ }^{-1-}$-BM-MSCs was much higher than Rap1 ${ }^{-1-}$-BM-MSCs (Figure 3dii and iii; $P<0.01$ ). These results further confirm that deletion of Rap1 increases the resistance of MSCs to the challenge of hypoxia.

\section{CdM of Rap1 ${ }^{-/-}$-BM-MSCs alleviates hypoxia-induced NCMC apoptosis}

The pro-inflammatory factors from Rap $1^{-/-}$-BM-MSCs and BMMSC-CdM under normoxic and hypoxic conditions were examined. As shown in Figure 4ai, ii and iii, compared with the normoxia-CdM (conditioned medium), the concentration of TNF- $a$, IL-6 and monocyte chemotactic protein 1 (MCP-1) was increased in hypoxia-CdM of Rap ${ }^{-1-}$-BM-MSCs and BM-MSCs. Furthermore, in the hypoxia-CdM, the concentration of TNF- $a$, IL- 6 and MCP-1 was much lower in the Rap1 $1^{-1-}$-BM-MSC group than in the BM-MSC group (Figure $4 a, P<0.01$ ). Notably, the concentration of TNF- $a$, IL-6 and MCP-1 was also increased in Rap1-Rap 1 ${ }^{-1-}$-BMMSC-hy-CdM compared with Rap1 ${ }^{-1-}-$ BM-MSC-hy-CdM, indicating that deletion of Rap1 reduces pro-inflammatory factor release by MSCs (Figure 4a).

Next, in order to examine the protective effects of MSC-hypoxiaCdM, neonatal cardiomyocytes (NCMCs) were divided into five groups and received the following different treatments: (1) control group; (2) $50 \mu \mathrm{l}$ serum- and antibiotic-free DMEM (Dulbecco's modified Eagle's medium; hypoxia group); (3) $50 \mu \mathrm{l}$ BM-MSCs

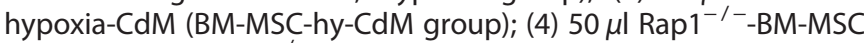
hypoxia-CdM (Rap1 ${ }^{-/-}-$BM-MSC-hy-CdM group); and (5) Rap1Rap1 ${ }^{-1-}$-BM-MSC hypoxia-CdM (Rap1-Rap1-1--BM-MSC-hy-CdM group) and then cultured for $48 \mathrm{~h}$ under hypoxia. Then terminal 

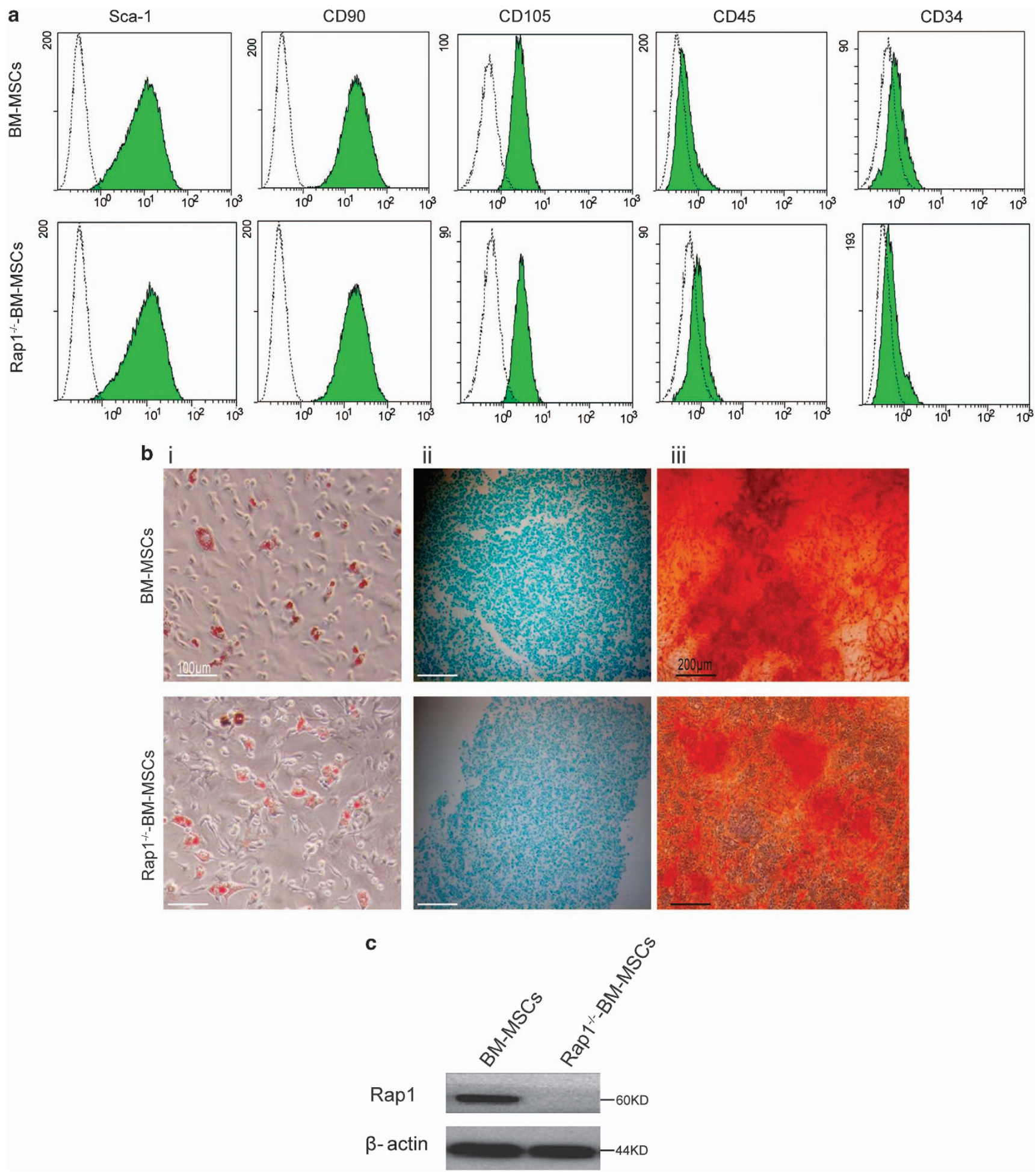

Figure 1. Characterization of BM-MSCs and Rap1 $1^{-1-}$-BM-MSCs. (a) Surface markers profiling determined with FASC that is positive for Sca-1, CD90 and CD105; negative for CD34 and CD45. (b) (i) Oil red staining for adipogenesis. (ii) Alcian blue staining for chondrogenesis. (iii) Alizarin red staining for osteogenesis. (c) Expression of Rap1 in BM-MSCs and Rap $1^{-1}-$-BM-MSCs.

deoxynucleotidal transferase-mediated dUTP nick end labeling (TUNEL) assay was performed to examine apoptosis in the different groups. As shown in Figures $4 \mathrm{~b}$ and $\mathrm{c}$, the apoptotic rate of NCMCs in the hypoxia group was dramatically increased compared with the control group $(P<0.01)$. In contrast, the apoptotic rate in Rap1 ${ }^{-/-}$-BM-MSC-hy-CdM-, BM-MSC-hy-CdM- and
Rap1-Rap1 1/--BM-MSC-hy-CdM-treated groups was significantly decreased compared with the hypoxia group $(P<0.01)$. In addition, the apoptotic rate in the Rap $1^{-/-}$-BM-MSC-hypoxiaCdM-treated group was lower than that in the BM-MSC-hypoxiaCdM-treated group $(P<0.01)$. Notably, the apoptotic rate was significantly increased in the Rap1-Rap $1^{-1-}$-BM-MSC-hy-CdM 
a

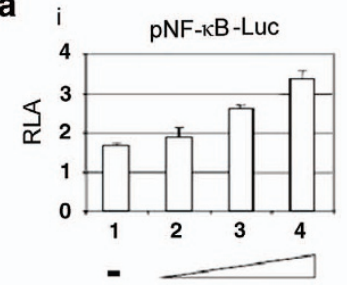

-

b
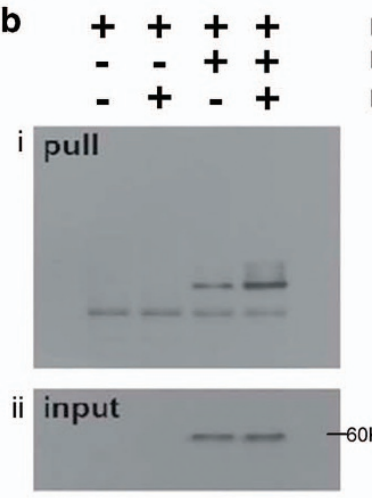

iii pull

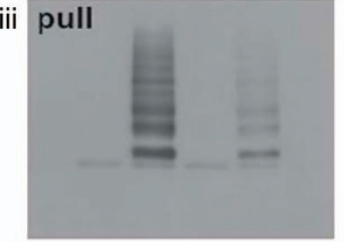

iv input

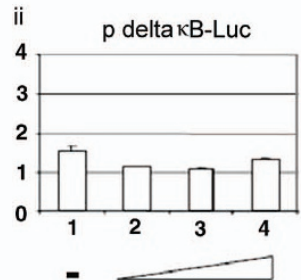

=

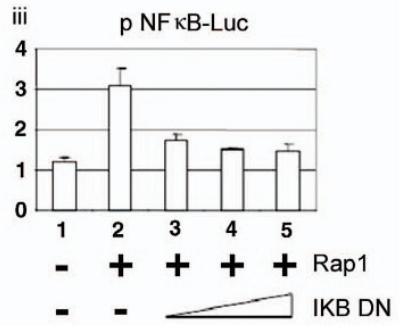

C
His-Ub

Rap1

IKK $\gamma$

$$
\text { - Ub-Rap1 }
$$

- ns

\section{Rap1}
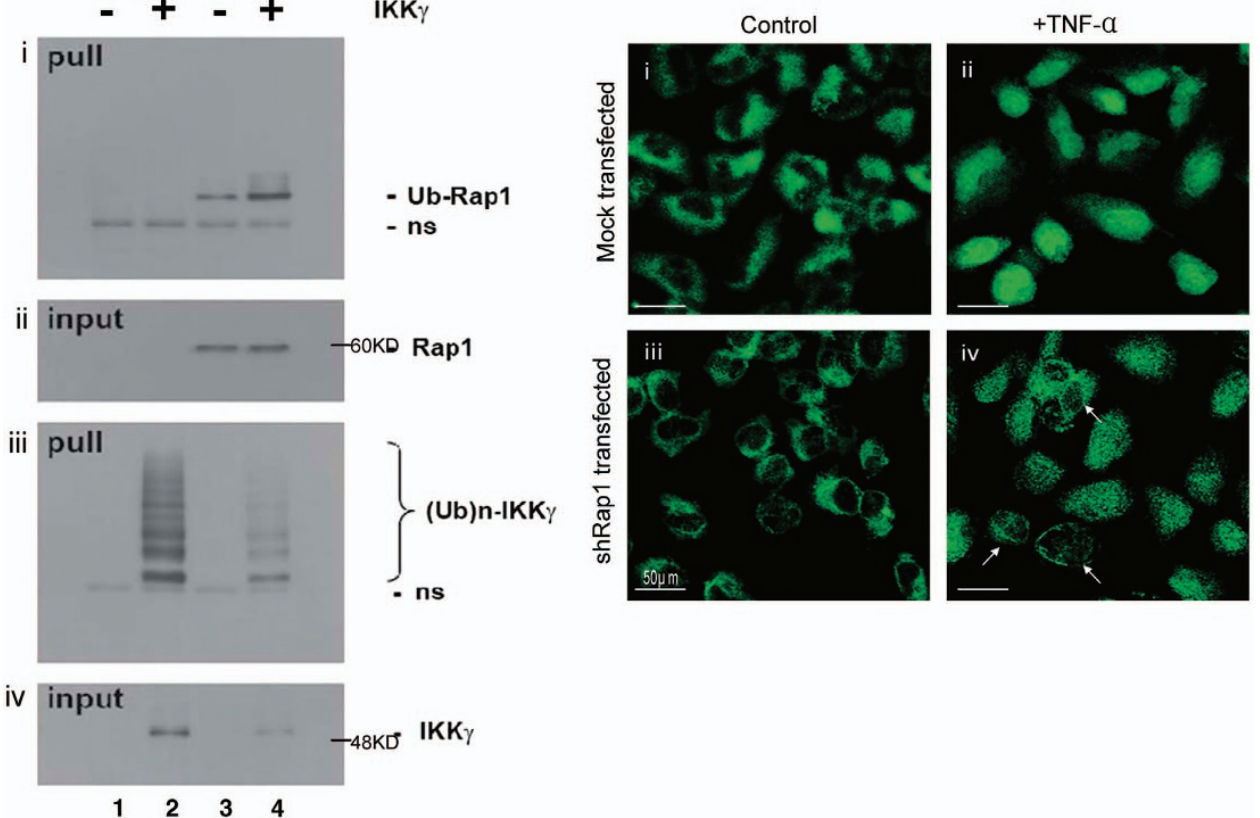

Figure 2. Activation of NF- $\kappa B$ transcriptional activity by Rap1. (a) HeLa cells were transfected with indicated plasmids. Groups i and iii received WT reporter constructs $p$ NF- $K B$-Luc, whereas group B received delta NF- $k B$-Luc. All groups received pSV-RLuc. The amounts of expression plasmid for Rap1 ( $\mathrm{i}$ and ii) and IkB-dominant-negative (iii) were progressively increased as indicated. Relative luciferase activity (RLA) represents firefly luciferase activity recovered from pNF- $\kappa B$-Luc or $\mathrm{p}$ delta $\kappa B$-Luc normalized to Renilla luciferase activity recovered from pSV-RLuc. (b) HEK293T cells were transfected with expression plasmids for Flag-tagged Rap1 (lanes 3 and 4) and HA-tagged IKKY (lanes 2 and 4). All groups received His-tagged ubiquitin expression plasmid. His-tagged ubiquitin conjugates were pulled down. The ubiquitinated Rap1 and IKK $\gamma$ was detected using western blot analysis with anti-Flag (i) and anti-HA (ii), respectively. The input lysates were also probed for anti-Flag and anti-HA to detect Rap1 and IKKY, respectively (ii and iv). (c) Representative photographs show the location of NF-KB-p65 in HeLa cells (i) and shRap1-transfected HeLa cells (iii). NF-kB-p65 was translocated from the cytoplasm to nuclei responding to TNF stimulation (ii). Knocking down Rap1 with shRap1 resulted in diminished translocation of NF-kB-p65 from the cytoplasm to nuclei responding to TNF stimulation (iv).

group compared with the Rap1 ${ }^{-1-}$-BM-MSC-hy-CdM group, indicating that increased Rap1 can downregulate the cardioprotection of Rap1 $1^{-/-}$-BM-MSCs by regulating the paracrine effects (Figures $4 \mathrm{~b}$ and $\mathrm{c}$ ).

Transplantation of Rap $1^{-/-}$-BM-MSCs enhances cell survival and cardioprotection in $\mathrm{Ml}$

Invasive hemodynamic measurement at 4 weeks post-cell transplantation showed that, compared with the Ml group, left ventricle end systolic pressure (LVESP), maximum dp/dt and slope of end systolic pressure volume relationship (ESPVR) were significantly increased (Figures $5 \mathrm{a}$ and $\mathrm{b} ; P<0.01$ ) in the MSC transplantation groups. Furthermore, LVESP, maximum $\mathrm{dp} / \mathrm{dt}$ and slope of ESPVR were significantly higher in the Rap1 $1^{-1-}-\mathrm{BM}-\mathrm{MSC}$ group than in the BMMSCs group (Figures $5 \mathrm{a}$ and bi, ii and iii; $P<0.01$ ).

Cardiac fibrosis was assessed using Masson Trichrome staining (Figure 5c). At 4 weeks post-MSC transplantation, cardiac fibrosis was significantly reduced by $28.1 \%$ in the BM-MSCs group and by
$68.2 \%$ in the Rap1 ${ }^{-1-}$-BM-MSCs group, compared with the MI group (Figure $5 \mathrm{~d} ; P<0.01$ ). Compared with the BM-MSCs group, the extent of cardiac fibrosis was also significantly lower in the Rap $1^{-/}-$BM-MSCs group (Figure 5d, 31.2 $\pm 5.4 \%$ versus $13.8 \pm 3.3 \% ; P<0.01)$.

Before cell transplantation, MSCs were labeled with Qtracker. The engrafted MSCs at 4 weeks post-transplantation in the ischemic myocardium were visualized and quantified. Both BMMSCs and Rap1 $1^{-1-}$-BM-MSCs were successfully engrafted in the ischemic heart tissue (Figure 5e). Notably, the number of Qtrackerpositive cells that survived in the ischemic myocardium 4 weeks posttransplantation was much higher in the Rap $1^{-1-}-\mathrm{BM}-\mathrm{MSC}$ group than in the BM-MSCs group (Figure 5fi, 36.6 $\pm 6.4 / 20 \times$ versus $16.6 \pm 4.5 / 20 \times ; P<0.01)$. Immunostaining with troponin showed that some Qtracker-positive cells in the ischemic myocardium differentiated into cardiomyocytes (Figure 5e). Nevertheless, only a small proportion of retained BM-MSCs $(\sim 4.9 \%)$ and Rap $1^{-1-}$-BM-MSCs ( 5.3\%) had transdifferentiation or cell fusion to cardiomyocytes (Figure $5 \mathrm{f}$ ii). 

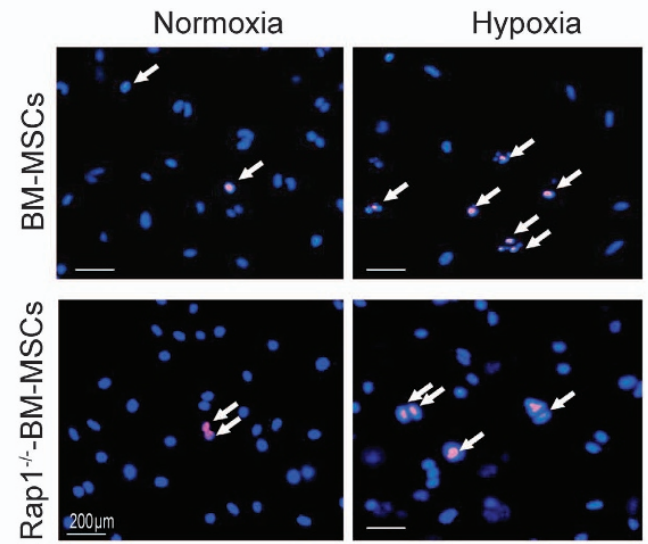

C

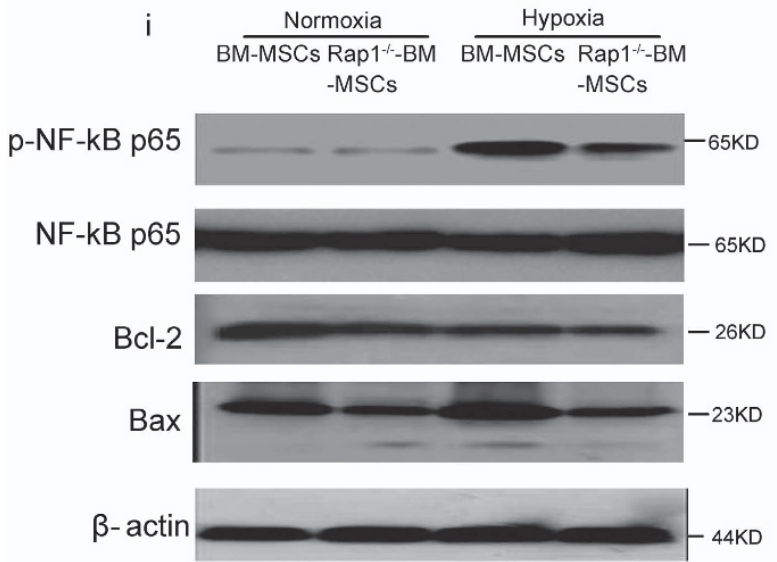

d

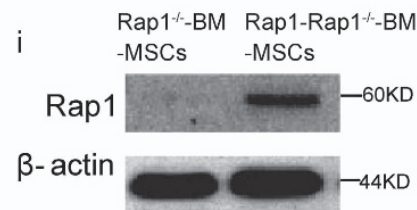

ii

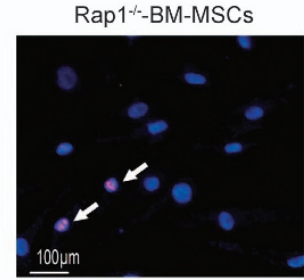

Rap1-Rap1 ${ }^{1-B M-M S C s}$

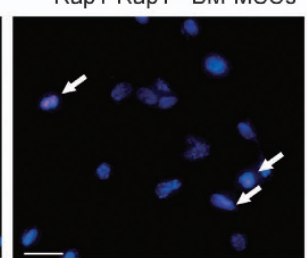

b
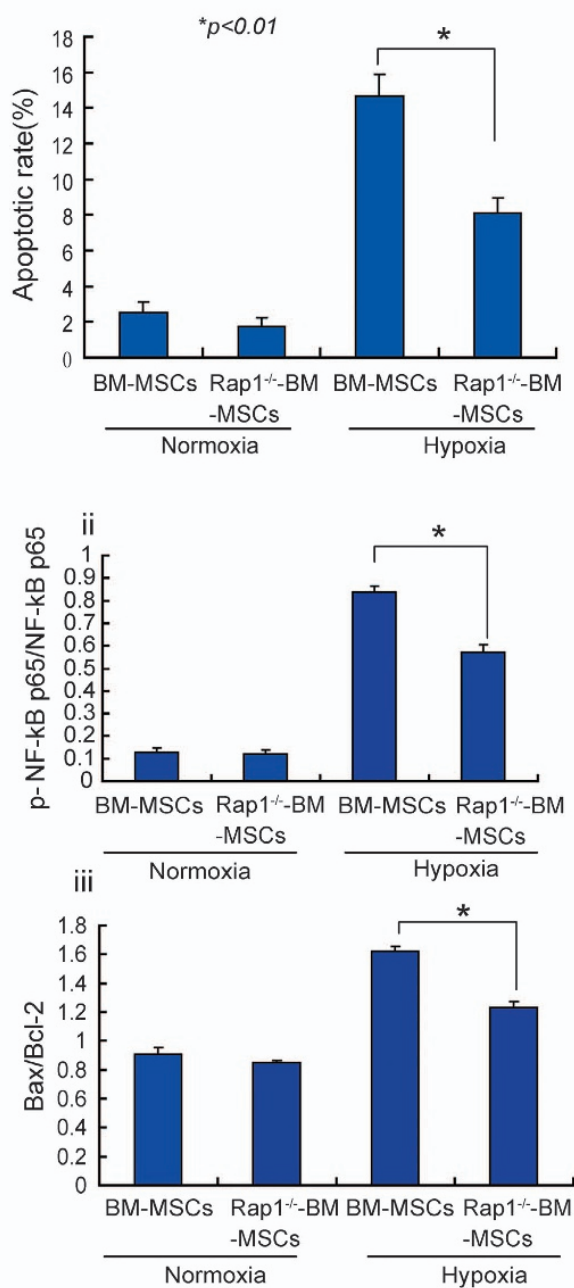

iii

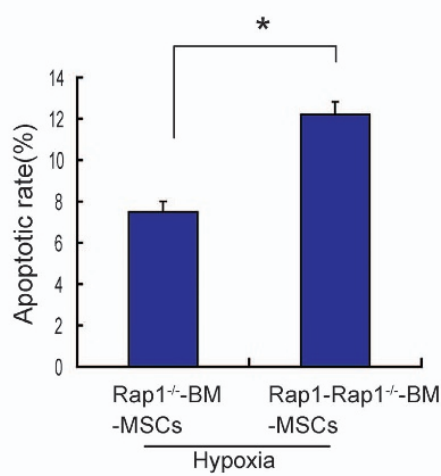

Figure 3. Rap1 $1^{-1-}$-BM-MSCs are more tolerant than BM-MSCs against hypoxia challenge. (a) Representative images show apoptosis of BM-MSCs and Rap1 $1^{-1-}$-BM-MSCs under normoxia and hypoxia. (b) The apoptotic rate was quantitatively measured in BM-MSCs and Rap1 ${ }^{-1-}-B M-M S C s$ under normoxia and hypoxia $\left({ }^{*} P<0.01\right)$. (c) (i) Western blot analysis shows the expression of $p-p 65-N F-k B$, p65-NF- $k B$, Bcl-2 and Bax of BM-MSCs and Rap1 ${ }^{-1-}-\mathrm{BM}-\mathrm{MSC}$ s under normoxia and hypoxia. (ii) Quantitative measurement of pNF- $\mathrm{BB}-\mathrm{p} 65 / \mathrm{NF}-\mathrm{kB}-\mathrm{p} 65$ of BM-MSCs and Rap1 ${ }^{-1-}$-BM-MSCs under normoxia and hypoxia after normalized to $\beta$-actin ( ${ }^{*} P<0.01$ ). (iii) Quantitative measurement of $\mathrm{Bax} / \mathrm{Bcl}-2$ of BM-MSCs and Rap1 ${ }^{-/-}$-BM-MSCs under normoxia and hypoxia $\left.{ }^{*} P<0.01\right)$. (d) (i) Western blot analysis shows overexpressed Rap1 in Rap $1^{-1-}$-BM-MSCs. (ii) Representative images show apoptosis of Rap $1^{-1}-$-BM-MSCs and Rap1-Rap $1^{-1-}-$ BM-MSCs under hypoxia. White arrows denote apoptotic cells. (iii) The apoptotic rate was quantitatively measured in Rap $1^{-/-}$-BM-MSCs and Rap1-Rap1 ${ }^{-/-}$-BM-MSCs under hypoxia $\left({ }^{*} P<0.01\right)$. All graphs represent the mean and error bars represent S.D. Data are representative of at least three independent experiments. 
Rap $1^{-/-}$-BM-MSCs transplantation reduces cardiomyocyte apoptosis linked to increased angiogenesis and decreased inflammation in vivo

As shown in Figures $6 a$ and $b$, compared with the control group, apoptosis of cardiomyocytes was significantly increased in the $\mathrm{Ml}$ group (Figures $6 a$ and $b, 23.3 \pm 3.3 \%$ versus $1.5 \pm 0.4 \%$; $P<0.01)$. Transplantation of BM-MSCs or Rap $1^{-/-}$-BM-MSCs significantly decreased the cardiomyocyte apoptotic rate (Figures $6 a$ and $b ; P<0.01$ ). In addition, the apoptotic rate in the Rap $^{-/-}$-BM-MSC group was much lower than in the a

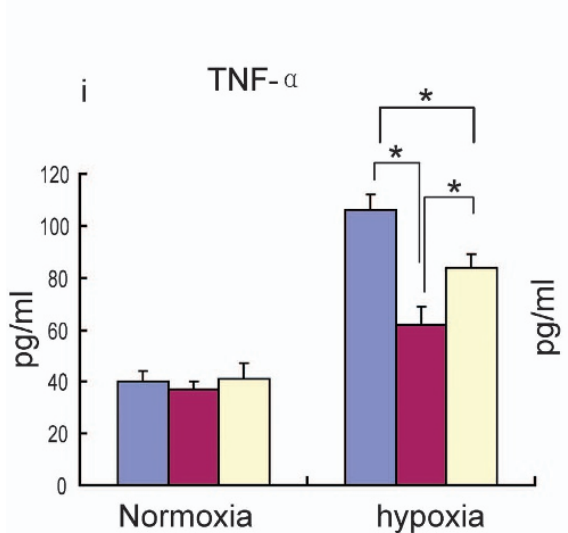

b

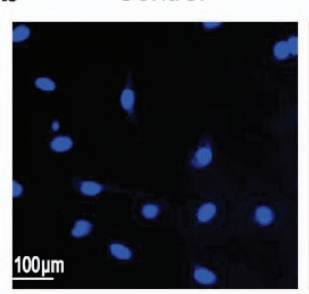

${ }^{*} p<0.01$

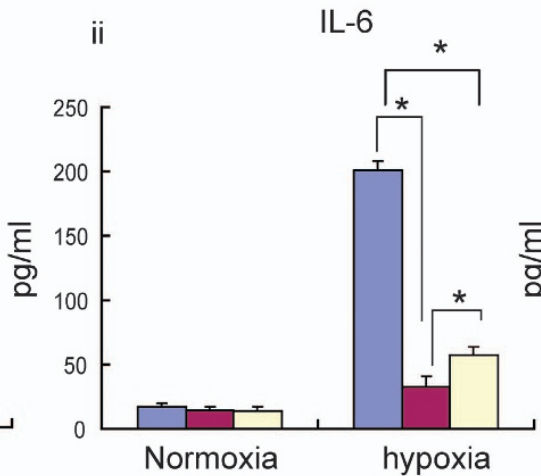

口 BM-MSCs

Rap ${ }^{1-\mathrm{B}} \mathrm{BM}-\mathrm{MSCs}$

$\square$ Rap1-Rap ${ }^{-1-B M-M S C s}$

iii

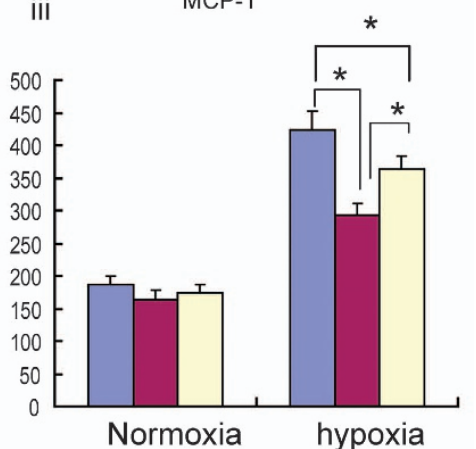

Hypoxia+BM-MSCs Hypoxia+Rap1--BM-MSCs Hypoxia+Rap1-Rap ${ }^{1-}$ -hy-CdM

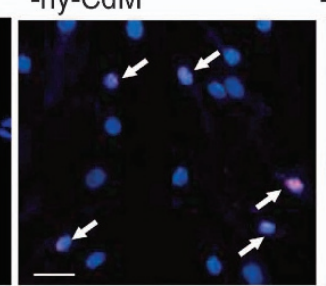
-hy-CdM
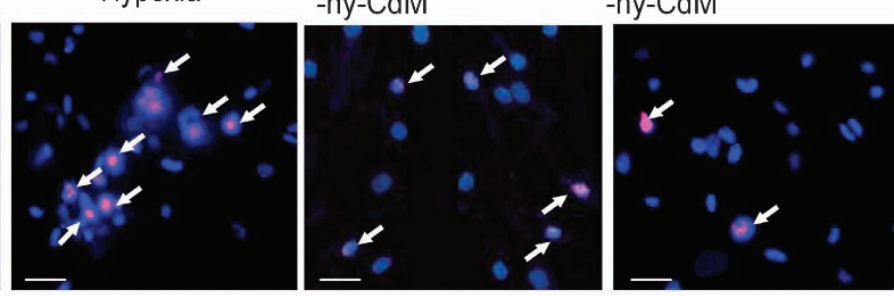

-BM-MSCs-hy-CdM

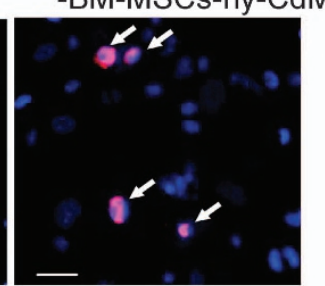

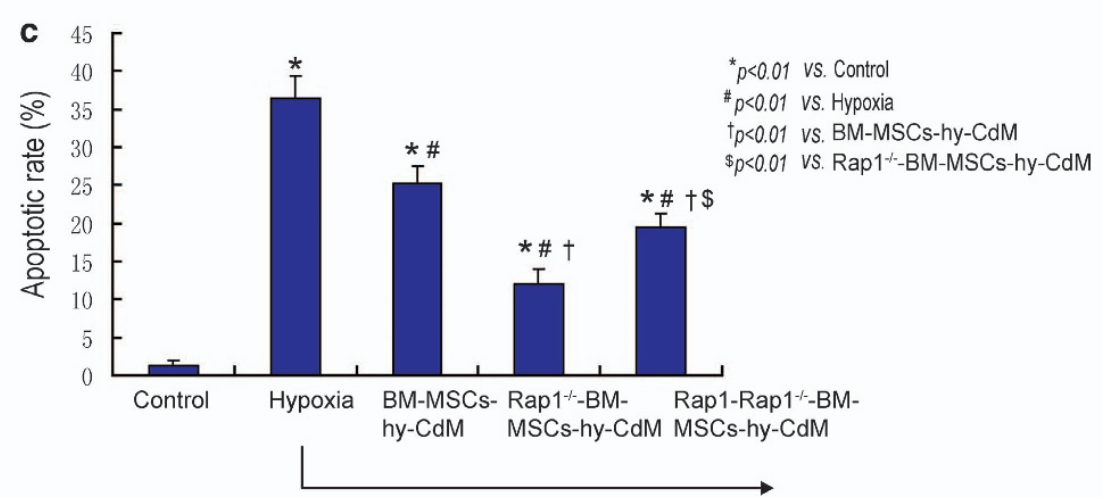

Figure 4. Distinct paracrine effects of BM-MSCs, Rap1-/--BM-MSCs and Rap1-Rap1-1--BM-MSCs. (a) The expression of TNF- $a$, IL- 6 and MCP-1 of BM-MSCs, Rap $1^{-1-}$-BM-MSCs and Rap1-Rap $1^{-1}$-BM-MSCs under normoxia and hypoxia $\left({ }^{*} P<0.01\right)$. (b) Representative images show apoptosis of NCMCs cocultured with the hypoxia-CdM from BM-MSCs, Rap $1^{-/}-$-BM-MSCs and Rap1-Rap 1 ${ }^{-1-}$-BM-MSCs under hypoxic condition. White arrows denote the apoptotic cells. (c) The apoptotic rate of NCMCs was measured among the different experimental groups $\left({ }^{*} P<0.01\right.$ versus the control group; ${ }^{\sharp} P<0.01$ versus the hypoxia group; ${ }^{\dagger} P<0.01$ versus the BM-MSCs-hy-CdM group; ${ }^{\$} P<0.01$ versus the Rap $^{-1-}$-BM-MSCs-hy-CdM group). All graphs represent the mean and error bars represent S.D. Data are representative of at least three independent experiments.

Figure 5. Rap1 $1^{-1-}$-BM-MSC transplantation restores heart function, reduces infarction size and increases cell survival in mice of the MI group. (a) Cardiac catheterization shows BM-MSC or Rap1-1--BM-MSC transplantation-restored LV function at 4 weeks after cell transplantation. (b) LVESP (i), +dp/dt (ii) and slope of ESPVR (iii) were quantitatively measured among the different experimental groups (*P $<0.01$ versus the control group; ${ }^{\#} P<0.01$ versus the Ml group; ${ }^{\dagger} P<0.01$ versus the BM-MSC group). (c) Representative images show at 4 weeks post-cell transplantation, MSC transplantation reduced the infarction size. (d) Fibrosis formation was measured in heart tissue among different experimental groups ( ${ }^{*} P<0.01$ versus the $\mathrm{MI}$ group; ${ }^{\#} P<0.01$ versus BM-MSC group). (e) At 4 weeks post-cell transplantation, Qtracker-positive cells in the heart tissue were detected in BM-MSC or Rap1-/--BM-MSC group. Moreover, some Qtracker-positive cells also coexpressed troponin, suggesting some grafted MSCs differentiated into cardiomyocytes. (f) (i) Qtracker-positive cell in the heart tissue was quantitatively measured in BM-MSC- or Rap $1^{-1-}$-BM-MSC-treated mice ${ }^{*} P<0.01$ versus BM-MSC group). (ii) The ratio of Qtracker-positive and troponinpositive cells to Qtracker-positive cell as percent of cell differentiation in BM-MSC- or Rap $1^{-{ }^{-}-}$-BM-MSC-treated mice was calculated. All graphs represent the mean and error bars represent S.D. Data are representative of at least three independent experiments. 
BM-MSC group (Figures $6 a$ and $b, 15.3 \pm 2.1 \%$ versus $8.8 \pm 1.5 \%$; $P<0.01)$.

The capillary density of the $\mathrm{Ml}$ area was evaluated by PECAM (CD31) staining. Compared with the control group, the capillary density was dramatically reduced in the $\mathrm{Ml}$ group (Figure $6 \mathrm{ci}$ and ii, $71.7 \pm 9.7 / 20 \times$ versus $19.6 \pm 7.9 / 20 \times ; P<0.01$ ). Transplantation of BM-MSCs or Rap $1^{-/-}$-BM-MSCs significantly increased the capillary density of the Ml area (Figure $6 \mathrm{ci}$ and ii; a

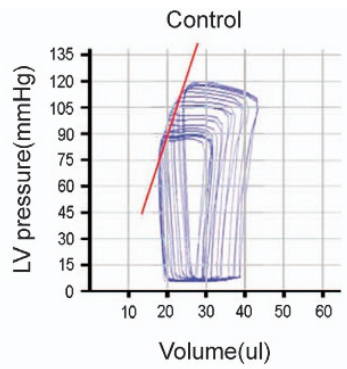

b

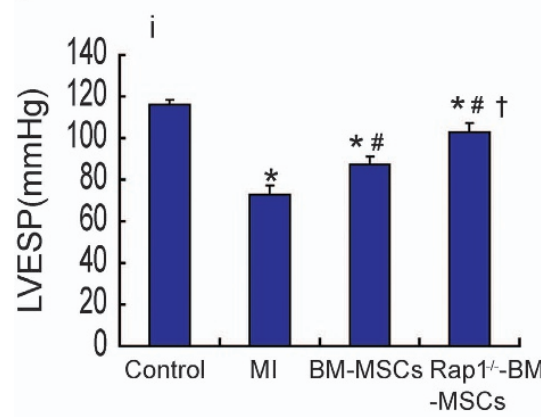

C
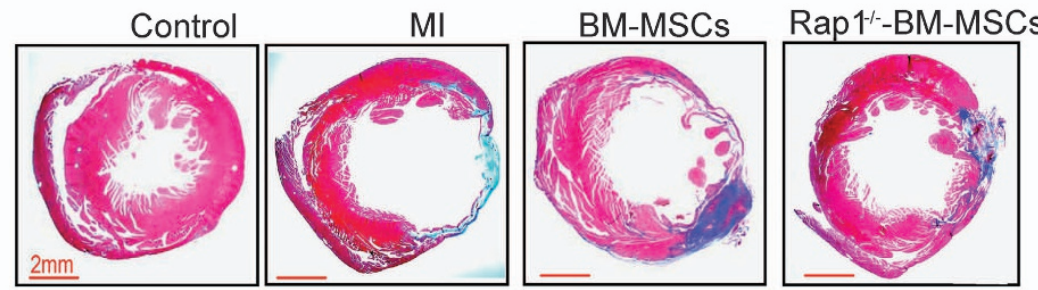

e
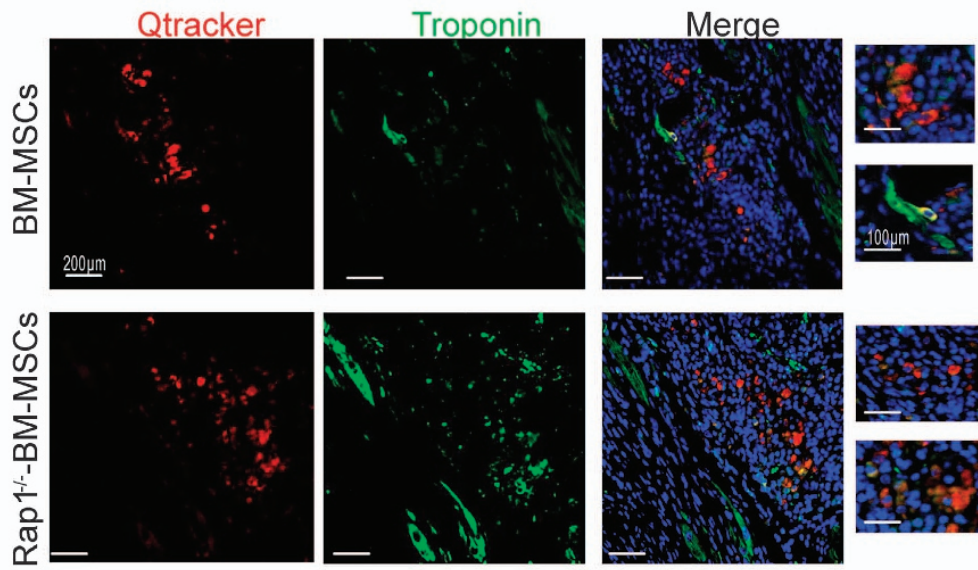

BM-MSCs

${ }^{*} p<0.01$ vs. Control

$\# p<0.01$ vs. MI

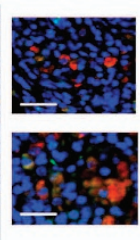

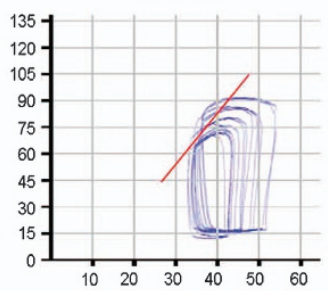
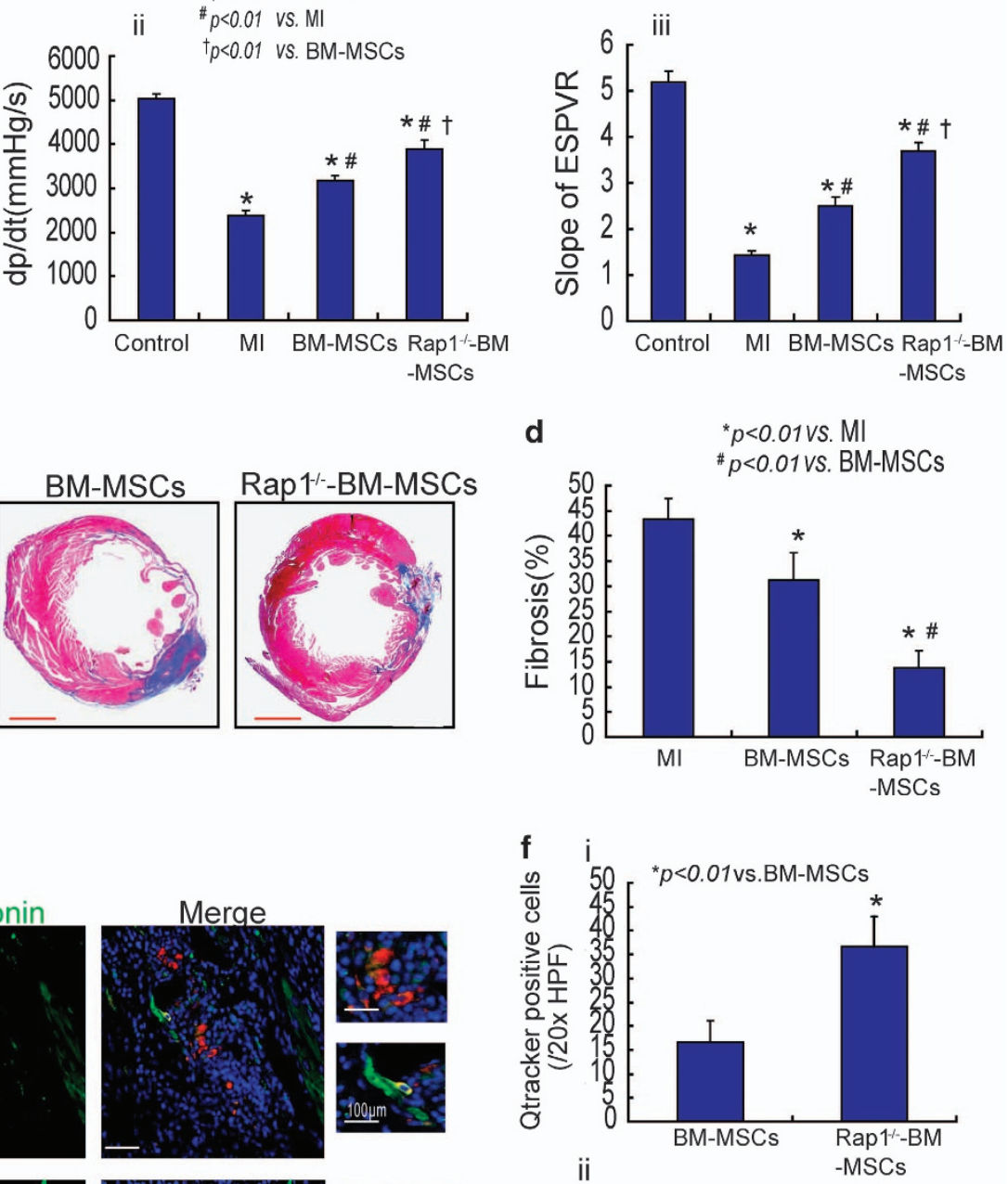

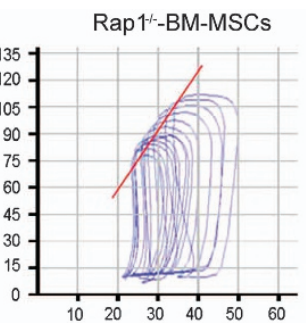
MSCs

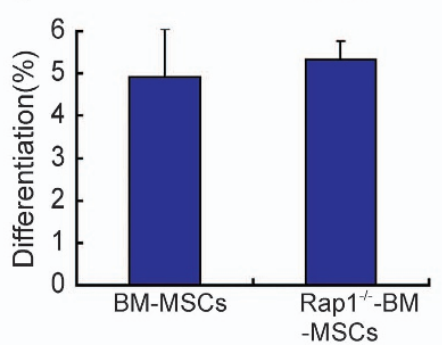


a
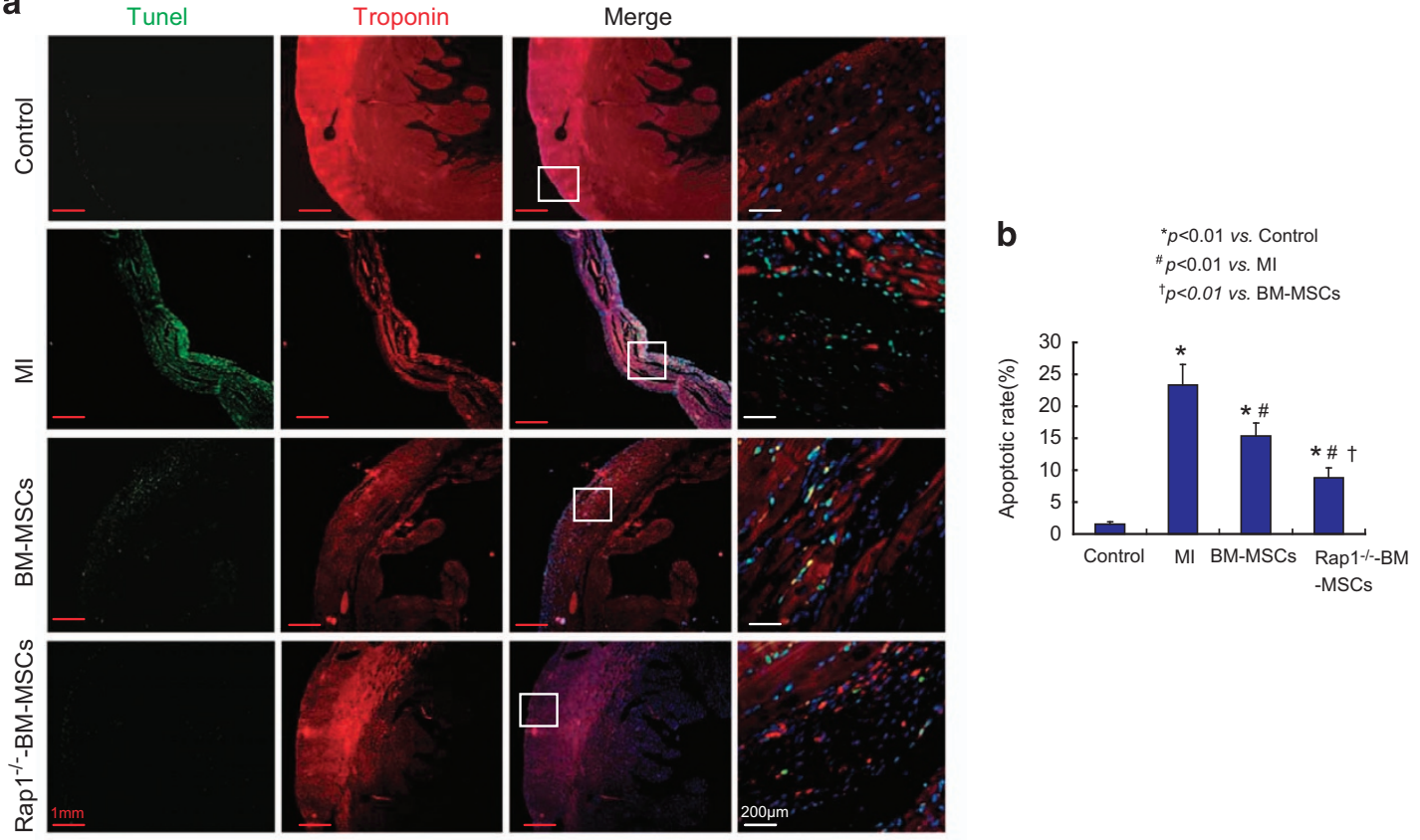

\section{c \\ i Control}
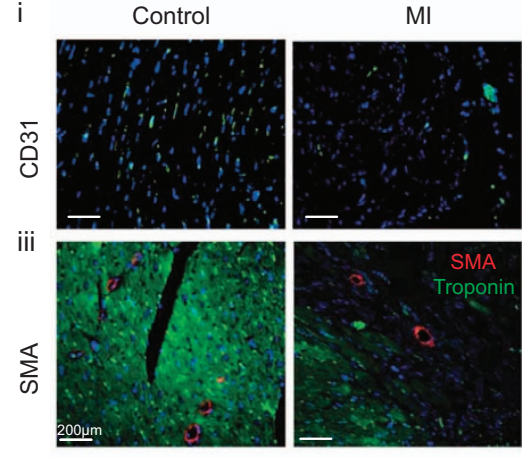

d
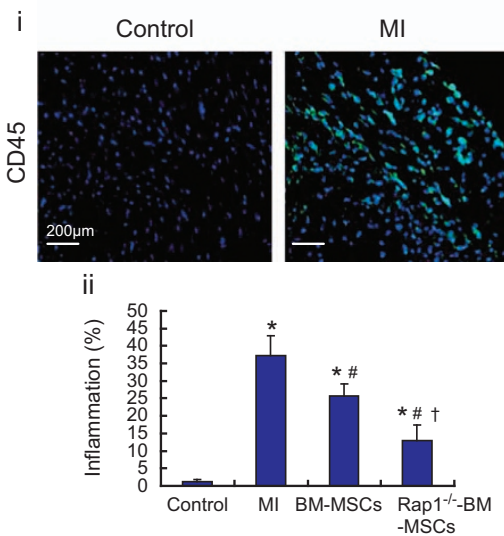

$\mathrm{Ml}$

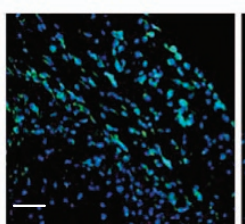

BM-MSCs

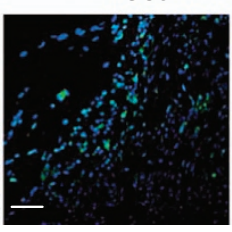

iii

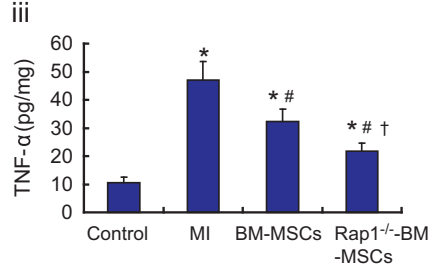

Rap1-/-BM-MSCs

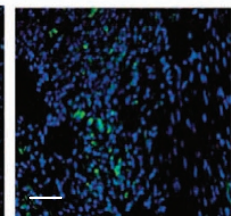

Rap $1^{-1-B M-M S C s}$
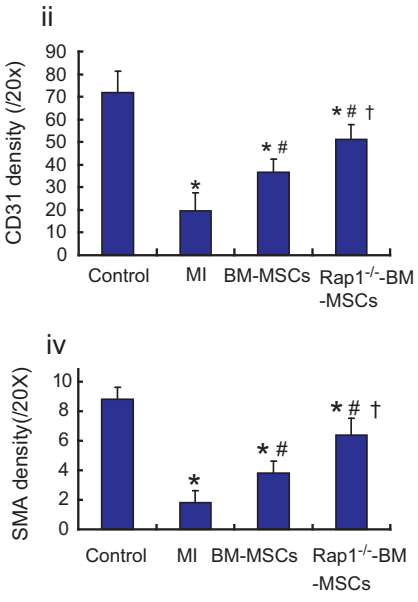

Figure 6. Rap $1^{-/-}$-BM-MSC transplantation ameliorates cardiomyocyte apoptosis, enhances angiogenesis and reduces inflammation in the mice of the Ml group. (a) Representative images show apoptosis of cardiomyocytes at 4 weeks post-cell transplantation in the heart tissue of MI. (b) The apoptotic rate was measured in heart tissue of different experimental groups. (c) Representative images show the capillaries (i) and blood vessels (iii) in the heart tissue at 4 weeks post-cell transplantation. (ii) The density of CD31 was measured in heart tissue among different experimental groups. (iv) The blood vessels in the heart tissue among the different groups were measured $\left({ }^{*} P<0.01\right.$ versus the control group; ${ }^{\#} P<0.01$ versus the Ml group; ${ }^{\dagger} P<0.01$ versus the BM-MSC group). (d) (i) Representative images show inflammatory infiltration mononuclearcyte CD45 cells accumulated in the heart tissue among different groups. (ii) The inflammation in the different groups was measured as percent of CD45-positive staining cells versus DAPI-positive cells per myocardium area. (iii) The concentration of TNF- $a$ among different groups was measured ( ${ }^{*} P<0.01$ versus the control group; ${ }^{\#} P<0.01$ versus the Ml group; ${ }^{\dagger} P<0.01$ versus the BM-MSCs group). All graphs represent the mean and error bars represent S.D. Data are representative of at least three independent experiments. 
$P<0.01$ ), and the capillary density in the Rap $1^{-/-}$-BM-MSC group was much higher than in the BM-MSC group (Figure $6 \mathrm{ci}$ and ii, $51 \pm 6.6 / 20 \times$ versus $36.7 \pm 5.8 \% ; P<0.01$ ).

The blood vessels of the $\mathrm{Ml}$ area were evaluated by smooth muscle actin (SMA) staining; blood vessels were greatly increased in the BM-MSC and Rap $1^{-1-}$-BM-MSC groups compared with the MI group (Figure 6 ciii and iv; $P<0.01$ ). Moreover, blood vessel density was much higher in the Rap $1^{-1-}$-BM-MSC group than in the BM-MSC group (Figure 6 ciii and iv, $6.4 \pm 1.1 / 20 \times$ versus $3.8 \pm 0.8 / 20 \times ; P<0.01)$.

One week post-cell transplantation, inflammation in heart tissue among the different experimental groups was evaluated by CD45 staining and ELISA. As shown in Figure 6di and ii, inflammation in the $\mathrm{Ml}$ group was dramatically increased compared with the control group (Figure $6 \mathrm{di}$ and ii, $37.3 \pm 5.6 \%$ versus $1.2 \pm 0.6 \%$; $P<0.01)$. Transplantation of BM-MSCs or Rap $1^{-1-}$-BM-MSCs significantly decreased this inflammation (Figure 6di and ii; $P<0.01)$. The inflammation in the Rap $1^{-1-}$-BM-MSC group was much lower than that in the BM-MSC group (Figure 6di and ii, $25.8 \pm 3.3 \%$ versus $13 \pm 4.5 \% ; P<0.01$ ).

Similar results were seen in the measurement of TNF- $a$ using ELISA in the different groups. Nonetheless, as shown in Figure 6diii, 1 week following MSC transplantation, TNF- $a$ was markedly reduced by 31.3 and $53.6 \%$ in the BM-MSC and Rap1 $1^{-1-}$-BM-MSCs groups, respectively, compared with the MI group (Figure $6 \mathrm{~d}$ and iii; $P<0.01$ ). In addition, TNF- $a$ in Rap1 $^{-1-}$-BM-MSCs was much lower than in BM-MSCs (Figure 6diii, $33.3 \pm 4.5$ versus $21.8 \pm 2.7 \mathrm{pg} / \mathrm{mg} ; P<0.01$ )

\section{DISCUSSION}

There are several major findings in this study. First, Rap $1^{-/-}$-BMMSCs were more tolerant than BM-MSCs to hypoxia that is associated with reduced activation of NF- $K B$ activity. Second, compared with BM-MSCs, Rap1 ${ }^{-1-}-\mathrm{BM}-\mathrm{MSC}$ showed a better therapeutic efficacy in MI. Third, Rap $1^{-1-}$-BM-MSC-mediated cardiac repair was associated with reduced inflammation post $\mathrm{MI}$ and enhanced cell survival. Finally, secretion profiling revealed reduced pro-inflammatory cytokines in Rap $1^{-1-}$-BM-MSCs such as TNF- $a, \mathrm{IL}-6$ and MCP-1.

NF- $K B$ has been documented as a pro- or anti-apoptotic gene that depends on the cellular context ${ }^{26-28}$ and which upstream signaling it is activated by. NF- $\kappa \mathrm{B}$ is also essential for the survival of many cells and functional response to stress. ${ }^{23}$ In endothelial cells, inhibition of NF- $K B$ activity significantly attenuates apoptosis induced by hypoxia. ${ }^{29}$ In this study, under hypoxic conditions, apoptosis of Rap $1^{-1-}$-BM-MSCs was much lower than that of BM-MSCs because of inhibition of NF-KB activity and Bax/BCl-2, and was confirmed using the western blot analysis. The Rap1 gene is a novel adaptor that regulates NF- $\kappa$ B activity by serving as the adaptor of the IKK complex, having a critical role in regulating cell apoptosis ${ }^{15}$ and metabolism. ${ }^{30}$ In this study, we showed that absence of Rap1 negatively regulated the activation of NF-KB in MSC. Our data also demonstrated that deletion of Rap1 in MSCs inhibits the activation of NF- $K \mathrm{~B}$, reduces the ratio of $\mathrm{Bax} / \mathrm{Bcl}-2$ and then directly attenuates the apoptosis induced by hypoxia, suggesting that inhibition of Rap1 enhances MSC survival capacity under hypoxia. These results prompt us to consider Rap1 ${ }^{-/}$-BM-MSC transplantation instead of BM-MSCs for MI in that Rap $1^{-/-}$-BM-MSCs have a stronger ability to survive in the hostile environment of the injured heart. In our in vivo study, we confirmed that 4 weeks after transplantation, more Rap $1^{-1-}-\mathrm{BM}$ MSCs than BM-MSCs survived and contributed more to the recovery of heart function.

NF- $K B$ activation enhances expression of pro-inflammatory cytokines such as IL- 6 , IL- 8 and TNF- $a$, and then provokes an excessive inflammatory response, with detrimental consequences. ${ }^{31,32}$ Total inhibition of NF-KB leads to cell functional defects. ${ }^{33}$
Selective inhibition of certain signals to reduce NF- $\kappa B$ activity has been proposed as a novel therapeutic strategy in many inflammatory diseases and cancer treatment. ${ }^{34,35}$ Previous studies have revealed that MSCs have contradictive effects with proand anti-inflammatory properties. ${ }^{36}$ Enhancement of the antiinflammatory effects of MSCs would be important to increase the efficacy of cardiovascular repair. ${ }^{37,38}$ Thus, deletion of Rap1 can regulate NF- $\kappa$ B activation, thereby mediating pro- and antiinflammatory cytokine release. Our data demonstrated that, under hypoxia, the expression of pro-inflammatory cytokines IL-6, TNF- $a$ and MCP-1 in the Rap1 ${ }^{-1-}$-BM-MSCs was much lower than in BMMSC CdM. This suggests that the absence of Rap1 reduces NF- $k B$ activation and decreases the pro-inflammatory cytokine production by MSCs. Furthermore, hypoxia-CdM from Rap $1^{-1-}-\mathrm{BM}-\mathrm{MSC}$ demonstrated a superior protective effect to BM-MSCs against myocardial death under hypoxia challenge, indicating that the beneficial paracrine function is enhanced in Rap $1^{-/-}$-BM-MSCs compared with WT MSCs. To further confirm the key role of Rap1 in regulating pro-inflammatory cytokine release by MSCs, we transfected Rap1-1--BM-MSCs with Rap1; when Rap1-1--BMMSCs overexpressed Rap1, the expression of IL-6, TNF- $a$ and MCP-1 was elevated under hypoxic conditions, suggesting that deletion of Rap1 has a strong capacity to regulate the paracrine action of MSCs.

There are some aspects of this study that need further investigation. First, the secretion of MSCs in the ischemic heart may differ under in vitro hypoxia. Second, apart from the antiinflammatory reaction of MSCs in this study, many other potential mechanisms of MSCs need to be further investigated. Third, whether deletion of Rap1 can influence other potential signaling pathways to mediate MSC-based therapy for cardiovascular disease also needs further investigation.

In summary, in this study we established that a novel NF- $K B$ adaptor, Rap1, has an important role in the regulation of MSC paracrine components and MSC capacity for survival in a stressful environment. Compared with WT BM-MSCs, transplantation of Rap $^{-1-}$-BM-MSCs achieved superior therapeutic efficacy in a mouse model of MI that may be attributed to Rap1-modulated cytokine bias and cell survival potential.

\section{MATERIALS AND METHODS}

\section{Mice}

The WT and Rap ${ }^{-1-}$ mice, provided by the National University of Singapore, were bred and kept in the Laboratory Animal Unit at the University of Hong Kong. All animal experiments in this study were approved by the Committee on the Use of Live Animals in Teaching and Research at The University of Hong Kong.

Isolation, culture and characterization of Rap $1^{-/-}-\mathrm{BM}-\mathrm{MSCS}$ and BM-MSCs

Rap $^{-1-}$-BM-MSCs and BM-MSCs were isolated from 6 8-week-old $\mathrm{Rap1}^{-1-}$ and WT mice, respectively. Briefly, following killing, femurs and tibiae were quickly removed from mice and bone marrow was harvested by strong flushing with DMEM (Invitrogen) supplemented with $10 \%$ fetal bovine serum (FBS, Gibco, USA) and P/S on ice. The resultant cell suspension was filtered using a 70-mm filter and bone marrow cells were planted in culture dishes with complete DMEM medium and incubated at $37^{\circ} \mathrm{C}$ with $5 \% \mathrm{CO}_{2}$ in a humidified chamber. Non-adherent cells were removed $3 \mathrm{~h}$ later by replacing the culture medium. Thereafter, the culture medium was replaced with fresh medium and the process repeated every $8 \mathrm{~h}$ until $72 \mathrm{~h}$ of initial culture was reached. MSCs were characterized using flow cytometry. Oil red staining was performed to identify adipocytes, alcian blue staining for chondrocytes and alizarin red staining for osteocytes. In order to track MSCs posttransplantation, MSCs were labeled with the cell tracker dye Qtracker 655 Cell Labeling Kit (Q25021MP, Life Technologies, Carlsbad, CA, USA) according to the manufacturer's instructions. The labeling efficiency was verified using flow cytometry. 
Preparation of CdM

The CdM of cultured Rap1-/--BM-MSCs and BM-MSCs was collected as previously reported. ${ }^{39}$ Briefly, MSCs were rypsinized and plated on a $10-\mathrm{cm}$ plate. After $24 \mathrm{~h}$, the completed culture medium was replaced with serumand antibiotic-free DMEM. Twenty-four hours later, serum-free cell culture supernatants were collected, filtered by a $0.22-\mu \mathrm{m}$ filter and centrifuged at $4{ }^{\circ} \mathrm{C}, 4000 \times g$ for $30 \mathrm{~min}$ using Amicon Ultra-4 Centrifugal Filter Devices (Millipore Billerica, MA, USA) to produce CdM. The final concentration was adjusted to $20 \times$ of collected CdM

\section{NCMC isolation and culture}

The NCMCs were isolated and cultured as described previously. ${ }^{40}$ Briefly, following killing of neonatal Wistar rats (0- to 1-day-old), the hearts were promptly removed, rinsed four times with modified Hank's solution and cut into small pieces on ice. The tissue fragments were transferred to a $50-\mathrm{ml}$ tube and warmed in a water bath with a magnetic bar for $10 \mathrm{~min}$ at $37^{\circ} \mathrm{C}$. After discarding the supernatant, the minced myocardium was digested with fresh pre-warmed $0.25 \%$ trypsin for $5 \mathrm{~min}$ at $37^{\circ} \mathrm{C}$, and the supernatant collected gently and transferred to a $50-\mathrm{ml}$ tube on ice containing $7 \mathrm{ml} \mathrm{FBS}$. These two steps were repeated to collect supernatant after which all supernatants were centrifuged at $156.8 \times \mathrm{g}$ for $5 \mathrm{~min}$ to collect the cells. Cells were re-suspended in NRVM culture medium to reduce fibroblast contamination. Finally, the supernatant was aspirated gently, and the cells were plated in MEA dishes at a density of $6 \times 0^{5}$ cells $/ \mathrm{ml}$. Culture medium was changed every day.

\section{TUNEL assay}

To directly visualize apoptosis of MSCs exposed to hypoxia and the NCMCs cocultured with MSC-CdM under hypoxic conditions, TUNEL assay was performed according to the manufacturer's instructions. Briefly, after washing with PBS, the cells on the cover slide were incubated with $1 \mu \mathrm{g} / \mathrm{ml}$ Proteinase $\mathrm{K} / 10 \mathrm{mM}$ Tris solution at room temperature for $15 \mathrm{~min}$ and then washed twice in PBS and finally incubated with $50 \mu \mathrm{l}$ TUNEL reaction mixture in a humid chamber for $1 \mathrm{~h}$ at room temperature. Cover slides were washed twice with PBS, mounted with 4, 6-diamidino-2-phenylindole (DAPI), observed under a fluorescent microscope and finally photographed.

\section{Western blot analysis}

Total proteins were extracted from cells using RIPA buffer and the concentration measured. Protein $(20 \mu \mathrm{g})$ was separated in a $10 \%$ SDS-PAGE gel and transferred on a PVDF membrane (Millipore). The membrane was washed three times in Tris-buffered saline (TBS) with $0.1 \%$ Tween-20 and blocked with $5 \%$ fat-free milk in TBS for $1 \mathrm{~h}$ at room temperature. Subsequently, the membrane was incubated with anti-Rap1, Bcl-2, Bax, p-NF-KB-p65 and NF-KB-p65 (Santa Cruz, Dallas, TX, USA; $1: 2000$ ) overnight at $4{ }^{\circ} \mathrm{C}$ followed by incubation with horseradish peroxidaseconjugated secondary antibodies (1:10000 dilution; Santa Cruz). Finally, the signal on the membranes was visualized using $\mathrm{ECL}$ and exposed to medical X-ray films for seconds or minutes.

\section{Lentiviral construct packaging and infection}

The lentivirus was packaged by co-transfecting 293T cells using the lentiviral packaging system comprising the recombinant lentiviral transfer Rap1 plasmid or shRap1 plasmid, packaging (GAG/Pol and REV) plasmids and envelope (VSV-G) plasmid. Following 48-h culture, the supernatant of transfecting 293T cells was collected, concentrated and tittered. Subsequently, the virus was used to infect Rap $1^{-1-}-$ BM-MSCs or HeLa cells.

\section{MI model and cell transplantation}

A MI model was induced in ICR mice (6 8-week-old). Mice were anesthetized with an intraperitoneal injection of ketamine $(100 \mathrm{mg} / \mathrm{kg})$ and xylazine $(20 \mathrm{mg} / \mathrm{kg})$ and connected to a ventilator via tracheal intubation. The heart was exposed via a left side limited thoracotomy and the middle of the left anterior descending artery (LAD) ligated with an 8-0 silk suture. At $60 \mathrm{~min}$ after induction of $\mathrm{Ml}$ mice were randomized to receive intramyocardial injection of (1) PBS (MI group, $n=12$ ); (2) $1.0 \times 10^{6}$ BM-MSCs (BM-MSC group, $n=13$ ); or (3) $1.0 \times 10^{6} \mathrm{Rap}^{-1-}$-BM-MSCs (Rap $1^{-1-}$-MSC group, $n=12$ ) at four sites in the border of the infarct area. Another group of mice $(n=10)$ underwent thoracotomy without LAD ligation and served as controls (Control group).

\section{Hemodynamic assessment}

At 4 weeks post-cell transplantation, mice were anesthetized and mechanically ventilated as described above. A 1.2-F pressure-volume conductance catheter connected to an ADVantage PV-Loop system (Scisence Inc., Ontario, Canada) for data acquisition was inserted into the LV cavity through the right carotid artery. Hemodynamic parameters, including LVESP and dp/dtmax, were recorded and analyzed using the LabScribe software (Scisence Inc.). The slope of the ESPVR was measured to evaluate heart function.

\section{Assessment of fibrosis and apoptosis}

Following hemodynamic assessment, mice were killed for histological and immunohistochemical study. Hearts were quickly taken out, washed with cold PBS and fixed in formalin for $24 \mathrm{~h}$. Hearts were then embedded in paraffin and cut into 5- $\mu \mathrm{m}$ sections from the apex, mid-LV. Fibrosis in the different experimental groups was analyzed using a Masson's Trichrome Stain Kit (HT15, Sigma, St. Louis, MO, USA). The percentage infarct size was calculated as (fibrosis area/total LVA) $\times 100 \%$. Apoptosis was evaluated with TUNEL staining as previously described.

\section{Immunohistochemical staining}

Immunohistochemical staining was performed according to the standard protocol. Following incubation with $5 \%$ bovine serum albumin for $30 \mathrm{~min}$, heart sections or cells were stained with primary antibody and incubated overnight at $4{ }^{\circ} \mathrm{C}$ with a $1: 100$ dilution. The antibodies were anti-Troponin (SC-8121, Santa Cruz), anti- $a$-SMA (SC-53142, Santa Cruz), anti-CD31 (SC-31054, Santa Cruz) and anti-CD45 (SC-25590, Santa Cruz). Sections were incubated with PBS instead of the primary antibody to serve as a negative control. Then, the second antibody with FITC-conjugated antimouse IgG $(1: 1000)$, anti-rat $\lg G(1: 1000)$ or anti-goat $\lg G(1: 1000)$ was added and incubated for $1 \mathrm{~h}$ at room temperature. Finally, heart sections were washed with PBS twice and mounted with 4, 6-diamidino-2phenylindole (DAPI) to stain the nucleus. Six mice from each group were analyzed; five sections were randomly collected from each mouse and then analyzed with a deconvoluted fluorescent microscope and Image $J$ software (National Institutes of Health, Bethesda, MD, USA).

\section{Statistical analysis}

Data are expressed as mean \pm S.D. The significant differences between groups were analyzed with unpaired Student's $t$-test for two groups or one-way ANOVA, followed by Bonferroni test for more than two groups. A value of $P<0.05$ was considered statistically significant.

\section{ABBREVIATIONS}

$\mathrm{BM}$, bone marrow; CdM, conditioned medium; ESPVR, end systolic pressure volume relationship; IL-6, interleukin-6; LVESP, left ventricle end systolic pressure; MCP-1, monocyte chemotactic protein 1; Ml, myocardial infarction; MSCs, mensenchymal stem cells; NCMCs, neonatal cardiomyocytes; NF-kB, nuclear factor-kappaB; TNF- $a$, tumor necrosis factor- $a$ (TNF- $a$ )

\section{ACKNOWLEDGEMENTS}

This research was supported by HKU Small Project Funding (201409176221 to YZ; 201007176100 to QL); Hong Kong Research Grant Council General Research Fund (HKU772510M to QL); State Key Laboratory of Pharmaceutical Biotechnology, The University of Hong Kong, to Professor Aimin Xu and QL; National Natural Science Grant of China, No 31270967 to QL) and Theme-based Research Scheme (T12-705/11 to $\mathrm{H}-\mathrm{FT}$ and $\mathrm{QL}$ ).

\section{COMPETING INTERESTS}

The authors declare no conflict of interest.

\section{REFERENCES}

1 Yousef M, Schannwell CM, Kostering M, Zeus T, Brehm M, Strauer BE. The BALANCE Study: clinical benefit and long-term outcome after intracoronary autologous bone marrow cell transplantation in patients with acute myocardial infarction. J Am Coll Cardiol 2009; 53: 2262-2269.

2 Schuleri KH, Boyle AJ, Hare JM. Mesenchymal stem cells for cardiac regenerative therapy. Handb Exp Pharmacol 2007; 180: 195-218. 
3 Gnecchi M, He H, Liang OD, Melo LG, Morello F, Mu H et al. Paracrine action accounts for marked protection of ischemic heart by Akt-modified mesenchymal stem cells. Nat Med 2005; 11: 367-368.

4 Amsalem Y, Mardor Y, Feinberg MS, Landa N, Miller L, Daniels D et al. Iron-oxide labeling and outcome of transplanted mesenchymal stem cells in the infarcted myocardium. Circulation 2007; 116: I38-145.

5 Muller-Ehmsen J, Krausgrill B, Burst V, Schenk K, Neisen UC, Fries JW et al. Effective engraftment but poor mid-term persistence of mononuclear and mesenchymal bone marrow cells in acute and chronic rat myocardial infarction. $J \mathrm{Mol}$ Cell Cardiol 2006; 41: 876-884.

6 Timmers L, Lim SK, Arslan F, Armstrong JS, Hoefer IE, Doevendans PA et al. Reduction of myocardial infarct size by human mesenchymal stem cell conditioned medium. Stem Cell Res 2007; 1: 129-137.

7 Schinkothe $T$, Bloch $W$, Schmidt A. In vitro secreting profile of human mesenchymal stem cells. Stem Cells Dev 2008; 17: 199-206.

8 Liu $\mathrm{CH}$, Hwang SM. Cytokine interactions in mesenchymal stem cells from cord blood. Cytokine 2005; 32: 270-279.

9 Uemura R, Xu M, Ahmad N, Ashraf M. Bone marrow stem cells prevent left ventricular remodeling of ischemic heart through paracrine signaling. Circ Res 2006; 98: 1414-1421.

10 Xu M, Uemura R, Dai Y, Wang Y, Pasha Z, Ashraf M. In vitro and in vivo effects of bone marrow stem cells on cardiac structure and function. J Mol Cell Cardiol 2007; 42: 441-448.

11 Mirotsou M, Zhang Z, Deb A, Zhang L, Gnecchi M, Noiseux N et al. Secreted frizzled related protein 2 (Sfrp2) is the key Akt-mesenchymal stem cell-released paracrine factor mediating myocardial survival and repair. Proc Natl Acad Sci USA 2007; 104: 1643-1648.

12 Burchfield JS, Iwasaki M, Koyanagi M, Urbich C, Rosenthal N, Zeiher AM et al. Interleukin-10 from transplanted bone marrow mononuclear cells contributes to cardiac protection after myocardial infarction. Circ Res 2008; 103: 203-211.

13 Kinnaird T, Stabile E, Burnett MS, Shou M, Lee CW, Barr S et al. Local delivery of marrow-derived stromal cells augments collateral perfusion through paracrine mechanisms. Circulation 2004; 109: 1543-1549.

14 Ward PA. The sepsis seesaw: seeking a heart salve. Nat Med 2009; 15: 497-498.

15 Teo $\mathrm{H}$, Ghosh S, Luesch H, Ghosh A, Wong ET, Malik N et al. Telomereindependent Rap1 is an IKK adaptor and regulates NF-kappaB-dependent gene expression. Nat Cell Biol 2010; 12: 758-767.

16 Ghosh AS, Tergaonkar V. Telomeres and inflammation: Rap1 joins the ends? Cell Cycle 2010; 9: 3834-3835.

$17 \mathrm{Li} \mathrm{Y}$, Tergaonkar V. Noncanonical functions of telomerase: implications in telomerase-targeted cancer therapies. Cancer Res 2014; 74: 1639-1644.

18 Low KC, Tergaonkar V. Telomerase: central regulator of all of the hallmarks of cancer. Trends Biochem Sci 2013; 38: 426-434.

19 Lee SJ, Jung YH, Oh SY, Song EJ, Choi SH, Han HJ. Vibrio vulnificus VvhA induces NF-kappaB-dependent mitochondrial cell death via lipid raft-mediated ROS production in intestinal epithelial cells. Cell Death Dis 2015; 6: 1655.

20 Wang Y, Wang GZ, Rabinovitch PS, Tabas I. Macrophage mitochondrial oxidative stress promotes atherosclerosis and nuclear factor-kappaB-mediated inflammation in macrophages. Circ Res 2014; 114: 421-433.

21 Cildir G, Akincilar SC, Tergaonkar V. Chronic adipose tissue inflammation: all immune cells on the stage. Trends Mol Med 2013; 19: 487-500.

22 Dey A, Wong E, Kua N, Teo HL, Tergaonkar V, Lane D. Hexamethylene bisacetamide (HMBA) simultaneously targets AKT and MAPK pathway and represses NF kappaB activity: implications for cancer therapy. Cell Cycle 2008; 7: 3759-3767.

23 Yang $\mathrm{K}$, Wang J, Xiang AP, Zhan X, Wang Y, Wu M et al. Functional RIG-l-like receptors control the survival of mesenchymal stem cells. Cell Death Dis 2013; 4: e967.
24 Mutt SJ, Karhu T, Lehtonen S, Lehenkari P, Carlberg C, Saarnio J et al. Inhibition of cytokine secretion from adipocytes by 1,25-dihydroxyvitamin $D(3)$ via the NF-kappaB pathway. FASEB J 2012; 26: 4400-4407.

25 Choi YH, Kurtz A, Stamm C. Mesenchymal stem cells for cardiac cell therapy. Hum Gene Ther 2011; 22: 3-17.

26 Basseres DS, Baldwin AS. Nuclear factor-kappaB and inhibitor of kappaB kinase pathways in oncogenic initiation and progression. Oncogene 2006; 25: 6817-6830.

27 Dhingra R, Shaw JA, Aviv Y, Kirshenbaum LA. Dichotomous actions of NF-kappaB signaling pathways in heart. J Cardiovasc Transl Res 2010; 3: 344-354.

28 Shin EM, Hay HS, Lee MH, Goh JN, Tan TZ, Sen YP et al. DEAD-box helicase DP103 defines metastatic potential of human breast cancers. J Clin Invest 2014; 124: 3807-3824.

29 Matsushita H, Morishita R, Nata T, Aoki M, Nakagami H, Taniyama Y et al. Hypoxiainduced endothelial apoptosis through nuclear factor-kappaB (NF-kappaB)mediated bcl-2 suppression: in vivo evidence of the importance of NF-kappaB in endothelial cell regulation. Circ Res 2000; 86: 974-981.

30 Tergaonkar V. p53 and NFkappaB: fresh breath in the cross talk. Cell Res 2009; 19: 1313-1315.

31 Libermann TA, Baltimore D. Activation of interleukin- 6 gene expression through the NF-kappa B transcription factor. Mol Cell Biol 1990; 10: 2327-2334.

32 Greenberg H, Ye X, Wilson D, Htoo AK, Hendersen T, Liu SF. Chronic intermittent hypoxia activates nuclear factor-kappaB in cardiovascular tissues in vivo. Biochem Biophys Res Commun 2006; 343: 591-596.

33 Poon MW, Yan L, Jiang D, Qin P, Tse HF, Wong IY et al. Inhibition of RAP1 enhances corneal recovery following alkali injury. Invest Ophthalmol Vis Sci 2015; 56: 711-721.

34 Barbie TU, Alexe G, Aref AR, Li S, Zhu Z, Zhang X et al. Targeting an IKBKE cytokine network impairs triple-negative breast cancer growth. J Clin Invest 2014; 124: 5411-5423.

35 Godefroy E, Gallois A, Idoyaga J, Merad M, Tung N, Monu N et al. Activation of toll-like receptor- 2 by endogenous matrix metalloproteinase-2 modulates dendritic-cell-mediated inflammatory responses. Cell Rep 2014; 9: 1856-1870.

36 English K. Mechanisms of mesenchymal stromal cell immunomodulation. Immunol Cell Biol 2013; 91: 19-26.

37 Du YY, Zhou SH, Zhou T, Su H, Pan HW, Du WH et al. Immuno-inflammatory regulation effect of mesenchymal stem cell transplantation in a rat model of myocardial infarction. Cytotherapy 2008; 10: 469-478.

38 Wen Z, Zheng S, Zhou C, Wang J, Wang T. Repair mechanisms of bone marrow mesenchymal stem cells in myocardial infarction. J Cell Mol Med 2011; 15: 1032-1043.

39 Zhang Y, Liao S, Yang M, Liang X, Poon MW, Wong CY et al. Improved cell survival and paracrine capacity of human embryonic stem cell-derived mesenchymal stem cells promote therapeutic potential for pulmonary arterial hypertension. Cell Transpl 2012; 21: 2225-2239.

40 Chan YC, Tse HF, Siu CW, Wang K, Li RA. Automaticity and conduction properties of bio-artificial pacemakers assessed in an in vitro monolayer model of neonatal rat ventricular myocytes. Europace 2010; 12: 1178-1187.

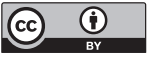

This work is licensed under a Creative Commons Attribution 4.0 International License. The images or other third party material in this article are included in the article's Creative Commons license, unless indicated otherwise in the credit line; if the material is not included under the Creative Commons license, users will need to obtain permission from the license holder to reproduce the material. To view a copy of this license, visit http://creativecommons.org/licenses/ by/4.0/ 